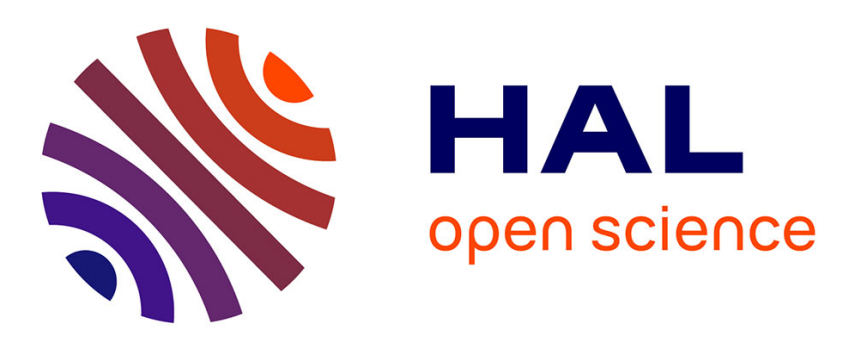

\title{
The Shaping of Dedekind's Rigorous Mathematics: What Do Dedekind's Drafts Tell Us about His Ideal of Rigor?
}

\author{
Emmylou Haffner
}

\section{To cite this version:}

Emmylou Haffner. The Shaping of Dedekind's Rigorous Mathematics: What Do Dedekind's Drafts Tell Us about His Ideal of Rigor?. Notre Dame Journal of Formal Logic, 2021, 62 (1), 10.1215/00294527-2021-0001 . halshs-03513278

\section{HAL Id: halshs-03513278 \\ https://shs.hal.science/halshs-03513278}

Submitted on 5 Jan 2022

HAL is a multi-disciplinary open access archive for the deposit and dissemination of scientific research documents, whether they are published or not. The documents may come from teaching and research institutions in France or abroad, or from public or private research centers.
L'archive ouverte pluridisciplinaire HAL, est destinée au dépôt et à la diffusion de documents scientifiques de niveau recherche, publiés ou non, émanant des établissements d'enseignement et de recherche français ou étrangers, des laboratoires publics ou privés. 


\title{
The Shaping of Dedekind's Rigorous Mathematics: What Do Dedekind's Drafts Tell Us About His Ideal of Rigor?
}

\author{
Emmylou Haffner
}

\section{Do not cite this version.}

\begin{abstract}
In this paper, I propose to examine Dedekind's ideal of rigor in the context of some of his mathematical drafts. After a presentation of his ideal of rigor based on statements in his published works, I use drafts from his Nachlass to study his invention of the new concept of Dualgruppe (equivalent to our modern lattice). I question the extent to which these preliminary researches hold up to the same standards of rigor. Focusing on a specific law of Dualgruppe theory, I show that the elaboration of a rigorous work can be the outcome of a process that is not necessarily so. I put forward the trial-and-error and inductive aspects of Dedekind's research practices. I consider whether the Dedekindian ideal of rigor guided mathematical research in its various phases, and what were consequences of such an ideal of rigor, if any, on mathematical research.

Keywords: 01 History of mathematics; 01A55 19th century; 06-03 Order, lattices, ordered algebraic structures: Historical; 00A30 Philosophy of mathematics.
\end{abstract}

\section{Introduction}

In a letter to Legendre from 12 April 1828, Jacobi answered to the latter's desire to know how he arrived at some of his results on the transformation of elliptic functions by stating the following:

$[\mathrm{T}]$ he route that $\mathrm{I}$ followed is not susceptible of any geometrical ${ }^{1}$ rigor. Once the discovery made, it would be possible to replace

\footnotetext{
${ }^{1}$ Recall that in the beginning of the 19th century, French speakers often used "geometer" for "mathematician" and "geometrical" for mathematical.
} 
it by a different route by which one can arrive to it rigorously. (...) Since all was confirmed by examples, I made bold to send a first letter to you, which you received with such graciousness. The proofs were only found later. [Jacobi, 1881, 415-416], transl. altered in [Grattan-Guinness, 2005, 422]

Jacobi recalled, in that letter, that it was through "happy trial and error" and by working on examples (which he generalized by induction) that he found his results. While this is certainly not an unusual statement for a mathematician, ${ }^{2}$ it does raise an interesting point about the shaping of rigorous mathematics: the process of finding a new result or a new concept can be quite non-rigorous, the rigor being instilled later, as a subsequent step of the mathematical research. And as a matter of fact, many considerations about rigor in mathematics consider questions of justification and/or verification of results, rather than their production.

While mathematicians can express very explicit and clear ideas about what rigorous mathematics should be, how it should be written, such statements tell us little about the various ways in which results are obtained. In fact, the ideals of rigor put forward by mathematicians might not necessarily reflect all practices of mathematical research, and could even hide some complex routes or phenomena that precede a step of rigorization. Without being so blunt (and naive) as to state that choices led by these ideals of rigor are solely choices about writing a publishable text, it is important to acknowledge that published mathematical texts are often taken as the paragon of mathematics, but are merely its front window. As Reuben Hersch suggested, mathematics has "a front and a back". ${ }^{3}$ Published texts and most mathematicians' public statements about their work show us the "front" of mathematics. In this paper, I would like to walk behind the scene for a case study on the shaping of a piece of mathematics by the German mathematician Richard Dedekind (1831-1916), who is considered an archetype of

\footnotetext{
${ }^{2}$ And Jacobi did not consider himself the most rigorous mathematician, stating in a 1846 letter to A. von Humboldt: "Only Dirichlet, not I, not Cauchy, not Gauss, knows what a perfectly rigorous proof is, but we learn it only from him. When Gauss says he has proved something, I think it very likely; when Cauchy says it, it is a fifty-fifty bet; when Dirichlet says it, it is certain; I prefer not go into these delicate matters" (Letter from 21 December 1846, quoted and translated in [Laugwitz, 2009, 63].

${ }^{3}$ [Hersch, 1991] draws on the anthropologist Erving Goffman's idea that institutions separate into the "front" and the "back": regions to which the public is admitted and from which it is excluded. Hersch suggests that "the 'front' of mathematics is mathematics in 'finished' form, as it is presented to the public in classrooms, textbooks, and journals. The 'back' would be mathematics as it appears among working mathematicians, in informal settings" [Hersch, 1991, 128].
} 
the rigorous mathematician. Using excerpts from his Nachlass, ${ }^{4}$ I propose to investigate the place held by rigor in the research process. I will consider whether Dedekind's statements about the criteria that make for rigorous mathematics are met by his research practices, as seen in his drafts.

To be clear, I will consider, here, Dedekind's own ideal of rigor, and will not be interested in evaluating rigor in terms of our modern standards. It is, indeed, important to acknowledge that rigor and ideals attached to it are context-dependent. As was underlined by A. D. Aleksandrov:

the rigor of maths is not absolute; it is in a process of continual development; the principles of maths have not congealed once and for all but have a life of their own and may even be the subject of scientific quarrels. [Aleksandrov, 1963]

History of mathematics provides many examples of statements, discussions and debates on rigor. Papers such as [Pierpont, 1928], [Kleiner, 1991] or [Hales, 2007] show the large variety of conceptions of rigor throughout history. It will not be my concern, here, to question Dedekind's ideal of rigor.

The characterization of Dedekindian rigor given so far relies only on published texts, whose writing was made for communication to the scientific community and does not always reflect the research process - a fact that was acknowledged by Dedekind himself. Yet, Dedekind often presents rigor as necessary for mathematical understanding. If this is indeed the case, some version of it should appear at least in part of his research process. Put bluntly, the question that led to this paper was the following: is Dedekind the same rigorous mathematician in his drafts as he is in his published papers? I observed elsewhere ${ }^{5}$ that his "conceptual" mathematics relied heavily on computations (and sometimes diagrams), and it seemed natural to wonder whether other aspects of what we think we know about Dedekind's mathematical practice could be shaken by studying his drafts.

Ideals of rigor have been an important interest of Mic Detlefsen's. ${ }^{6} \mathrm{He}$ has developed fine-grained, insightful analyses of ideas and ideals about rigor

\footnotetext{
${ }^{4}$ Cod. Ms. R. Dedekind, Niedersächsische Staats- und Universitätsbibliothek Göttingen.

${ }^{5}$ [Haffner, 2019]. [Edwards, 2010] makes a similar point on what he calls Riemann's algorithmic mathematics.

${ }^{6}$ As a matter of fact, rigor was the theme of the 2019 edition of the Philmath Intersem, co-organised by Notre Dame University and the Universite Paris Diderot.

Let me take the opportunity of this footnote to say a little more on Mic, Paris, and rigor. From 2007 to 2011, Mic Detlefsen held the Chaire d'Excellence Ideals of proof, from the French Agence Nationale de la Recherche with the Université Paris Diderot, Paris 1 Panthéon Sorbonne and the Université de Lorraine. The program was coming to an end as I was starting my PhD at the Université Paris Diderot. Jean-Jacques Szczeciniarz
} 
in several cases drawn from the history of mathematics, such as Bolzano's, Pasch's, Hilbert's, Weyl's, and Dedekind's. In a first section, I will expand on his analysis of Dedekind's ideal of rigor and clarify said ideal and its role in Dedekind's published works. In a second section, using Dedekind's drafts, I will consider whether the Dedekindian ideal of rigor described by Detlefsen holds throughout Dedekind's preliminary researches. This will raise an additional consideration: to what extent does rigor support or guide mathematical research in its various phases? What are some consequences of such an ideal of rigor, if any, on mathematical research? On one hand, we will see that Dedekind's research process relies largely on what appears to be systematic mathematical experimentations and inductive reasoning, which goes against the characterization of Dedekindian rigor. On the other hand, whereas a mathematician such as Felix Klein considered that rigor and mathematical creativity cannot coexist, ${ }^{7}$ it will also be the occasion to illustrate the extent to which the quest for rigorous mathematics can be a creative one. For this, I will use manuscripts leading to Dedekind's invention of a new concept, Dualgruppe, equivalent to our modern lattice.

suggested that I met with Mic to discuss my research project and whether this could be a good fit for a "co-advisorship" between Mic and Karine Chemla. "Yes, Mic said, I have a lot of ongoing projects on Dedekind." And this is how I became his student. Mic was fortunately very patient with my poor English and philosophy skills. I learned a lot at his side, and am incredibly grateful that he trusted the shy, impressionable student I was back then.

He also trusted me, a couple of years later, with working with him to organize the Philmath Intersem, a yearly 4-weeks long seminar co-organized by Paris, Nancy and Notre Dame as a follow up of the Ideals of proof activities. I became his right hand, and we worked together on the Intersem for eight years. Certainly our shared love for the good food and good wine that punctuated our Intersem meetings allowed our relationship to grow beyond our common appreciation of Dedekindian mathematics.

The Philmath Intersem was a successful event for ten years, and as David Rabouin and I secured new fundings in France, we were planning for it to continue at least until 2024. I know that I do not speak only for myself, but also for my colleagues in Paris and Nancy, when I say that (despite the technical difficulties brought by the events of 2020) we hope to continue this collaboration, which we consider to be a legacy from Mic.

Rigor - and a commentary on Mic's ideas on Dedekindian rigor - was the subject of my first published paper (in a special issue of Philosophia Scientice on rigor in mathematical practice, another continuation of the IP program). Rigor also appeared in my $\mathrm{PhD}$ dissertation, but maybe more than it should have. On a rainy afternoon, in his Notre Dame office, Mic told me "You cannot throw around the word rigor like that, especially not in the temple of Dedekind!". Several years later, when I first mentioned the ideas presented in this paper to him, Mic dared me to write this paper. I hope he would have been at least intrigued by the findings in Dedekind's Nachlass.

${ }^{7}$ In [Klein, 1895, 234], he states that "mathematics was by no means created through logical deduction". 
Dualgruppen were, according to Dedekind himself, the result of two decades of researches. We are lucky that these researches are available in Dedekind's Nachlass and that we can study the genesis of Dualgruppen. ${ }^{8}$

\section{Dedekindian rigor?}

\subsection{Rigor in Dedekind's published works}

Concerns with rigor appear central in many of Richard Dedekind's works. It was indeed what gave the impetus for his famous essay on irrational numbers published in 1872:

I found myself for the first time obliged to lecture upon the elements of the differential calculus; I felt more keenly than ever before the lack of a truly scientific foundation for arithmetic. In discussing the concept of the approach of a variable magnitude to a fixed limiting value - in particular, in proving the theorem that every magnitude which grows continually, but not beyond all limits, must certainly approach a limiting value - I took refuge in geometrical evidence. (...) For myself this feeling of dissatisfaction was so overpowering that I resolved to meditate on the question until I should find a purely arithmetical and perfectly rigorous foundation [Begründung] for the principles of infinitesimal analysis. [Dedekind, 1872, 767]

The introduction of the notion of cut to define irrational numbers was thus rooted in a desire to provide a rigorous foundation to analysis, that is, to mathematical investigations relying on the linear continuum. For Dedekind, to give such a rigorous foundation meant the following:

- Firstly, to proceed to a conceptual analysis so as to identify the necessary and sufficient conditions of a given property (or theory) - here, the continuity of the line.

- Secondly, to provide a definition for the irrational numbers and the linear continuum, based on said conceptual analysis.

- Finally, using the tools introduced for the definition of irrational numbers, to give a new definition for the operations in the extended system

\footnotetext{
${ }^{8}$ Cod. Ms. R. Dedekind III 14, X 9, X 10, X 11-1, X 11-2, XI 1, XI 2, XI 3-1 and XI $3-2$.
} 
of numbers, and for fundamental concepts of infinitesimal analysis such as the concept of limit.

Having laid this new rigorous foundation for real numbers allows, according to Dedekind, to give actual rigorous proofs of results such as $\sqrt{2} \times \sqrt{3}=\sqrt{6}{ }^{9}$ or of statements such as the Cauchy convergence test.

An explicit desire to develop a more rigorous foundation to a given theory was also expressed in Dedekind and Weber's 1882 paper on algebraic functions. In the introduction of their paper, they stated their desire to give a simpler, more general and more rigorous foundation to Riemann's theory of functions:

The purpose of the following investigations is to construct the theory of algebraic functions of one variable, which is one of Riemann's great creations, on the basis of a simple, yet at the same time rigorous and completely general viewpoint. [Dedekind and Weber, 1882, $41]$

Their work stemmed from their dissatisfaction with previous works on algebraic function theory which they saw as having tendencies to make "certain restrictive assumptions about the singularities of the functions", to treat the "so-called exceptional cases" either "casually as limiting cases" or not at all, or again to rely on "the evidence of geometric intuition" [Dedekind and Weber, 1882, 41]. All these were, for Dedekind and Weber, infringements to rigor.

Dedekind and Weber's paper relies on the transfer of Dedekind's numbertheoretical methods to fields of algebraic functions. In the first part, they study the theory of ideals in a field of algebraic functions of one complex variable. The second part consists in defining the Riemann surface, some of the related concepts (for example, its genus) and proving known results such as the Riemann-Roch theorem. For this, they start by defining the point of a Riemann surface as a mapping between $\mathbb{C} \cup \infty$ and the functions of the field previously studied. Then, they prove that there exists a oneto-one correspondence between points of the Riemann surface thus defined and ideals. This is the basis for their entire work, which thus establishes the foundation of Riemann surfaces in ideal theory, a purely arithmeticoalgebraic foundation. ${ }^{10}$

\footnotetext{
${ }^{9}$ Letter to Lipschitz, 10 June 1876, in [Dedekind, 1932, III, 468-474].

${ }^{10}$ For more on this see [Stillwell, 2012, Haffner, 2017].
} 
Dedekind and Weber considered their approach to be - at that point the only one that allows the resolution of the foundational difficulties faced by Riemannian function theory:

[I]t is known that a rigorous foundation of Riemann's theory presents certain difficulties and, until these have been completely overcome, it may be that the path we have taken, or at least something similar, is the only one leading to the goal with satisfactory rigor and generality. (...) Our work (...) takes a long algebraic detour through the theory of ideals, leading to a completely precise and rigorous definition of a "point of a Riemann surface" that can also serve as a basis for the investigation of continuity and related questions. [Dedekind and Weber, 1882, $42-43]$

Their assessment comes from the following. Firstly, their work does not rely on any kind of intuition or so-called evidence (geometric or other). This was also an argument in favor of giving a purely arithmetical definition of irrational numbers in [Dedekind, 1872]. In [Detlefsen, 2012], Detlefsen stresses this point as a central element of Dedekind's ideal of rigor:

[Dedekind] believed that judgments of immediacy were often problematic. In particular, he believed they are often made in ignorance of the sometimes elaborate reasonings by which socalled "immediate" judgments are originally acquired. (...) To one taking such a view, it was only natural that appeals to intuition should be seen as promoting the underestimation of the justificative complexity of arithmetical beliefs. [Detlefsen, 2012, 206-207]

Secondly, since their theory uses a general concept of algebraic function of one complex variable and relies on the theory of ideals of such functions - that is, sets of functions defined and studied without taking into account the individual nature of the elements -, it avoids any a priori hypotheses on the singularities of functions. Rather, the singularities are defined and studied in the new ideal-theoretic framework. This allows them to ensure that the theory covers all cases, thus avoiding the pitfalls of developing the theory on the basis of particular properties (only valid for certain functions) and of having special cases escaping the theory. ${ }^{11}$

\footnotetext{
${ }^{11}$ Dedekind's insistence on not basing the definitions on Darstellungformen, such as development of functions in series, falls in that category.
} 
Thirdly, for the same reasons, their theory also avoids using any undefined or ill-defined notion, since everything needed is (re-)defined or (re)proved carefully in the algebraic framework of ideal theory. The theory thus relies on well-defined concepts and well-proved results, thus avoiding both surreptitious assumptions (for example, assumptions on the continuity of functions) and gaps in deductions.

This is related to an important principle of Dedekind's ideal of rigor stated in the preface of Was sind und was sollen die Zahlen?:

In science nothing capable of proof ought to be believed without proof. [Dedekind, 1888, 790, transl. slightly altered]

In [Detlefsen, 2012], Detlefsen takes the opening statement of [Dedekind, 1888] as a core statement of Dedekind's ideal of rigor, stating that:

The only proper, and the best possible scientific justification of a provable proposition is a proof. More accurately, it is a proof in which all the basic premises are unprovable. [Detlefsen, 2012, 210]

This principle, which he calls "Dedekind's principle", gives us a standard of rigor for mathematics, according to which a proof (or definition) can only be considered a rigorous - and thus acceptable - one if it relies on unprovable premisses. This goes with the idea that a proof or definition should verify a justificative order or hierarchy.

[Dedekind, 1888] is thought of as an illustration, or even a realization, of that motto. Having observed the lack of rigorous proofs at the foundation of the arithmetic of natural numbers, Dedekind set himself the task of defining the set of natural numbers using solely the notions of set and mappings - the mathematical tools conceptualizing the operations and laws of thought, insofar as arithmetic flows "directly from the pure laws of thought" [Dedekind, 1888, transl. altered, 791]. As he wrote to Keferstein, on February 27 1890, Dedekind's aim was to

subsume [the mutually independent fundamental properties of the sequence of natural numbers] under more general notions and such activities of the understanding without which no thinking is possible at all but with which a foundation is provided for the reliability and completeness of proofs and for the construction of consistent notions and definitions. [Heijenoort, 1967, 99-100] 
Thus "divested of their specifically arithmetic character" [Heijenoort, 1967, 100], the properties of the set of natural numbers can be given with a purely set-theoretical (in Dedekind's terms, "logical") definition.

Dedekind's essay on the natural numbers is driven by similar principles as the ones I described above: to analyze the concept (here, the natural numbers) so as to identify its fundamental properties, to identify the right order of definition and proof to ground said concept (for example, Dedekind defined what it meant for a system to be infinite before defining the system of natural numbers), ${ }^{12}$ to give definitions and proofs relying on earlier fundamental concepts (here, sets and mappings), and finally to use this basis to re-define notions and/or re-prove results (for example, the four arithmetical operations and their properties). Dedekind chose to define a "simply infinite system", that is, an infinite countable totally ordered set - a general definition of a concept of which the set of natural numbers is an instance. By doing so, he very careful avoided putting any "arithmetical" property, that is, any property of the natural numbers, at the foundation of his definition of the set of natural numbers. He thus avoided any circular reasoning and any illicit assumption of existence. The choice of a set-theoretical definition is also a way to satisfy the statement opening [Dedekind, 1888], as the laws of thought were commonly thought of as being the only objectively unprovable truths. ${ }^{13}$

In all of these examples of applications of Dedekindian rigor presented here, we observe that Dedekind's emphasis is on providing a definition of given fundamental concepts, such as natural and real numbers, or the Riemann surface (indeed fundamental for Riemannian function theory). Hourya Benis-Sinaceur hasextent suggested that in Dedekind's principle, "capable of proof" can be taken as synonymous for "capable of definition": ${ }^{14}$

Dedekind could have written as well: "nothing capable of definition ought to be believed without definition". Otherwise, proofs can contain holes, be circular, or impossible. This approach takes him away from his contemporaries as well as from future con-

\footnotetext{
${ }^{12}$ Indeed, Dedekind wrote to Keferstein that his essay "came into being (...) [s] urely not all at once, rather it is a synthesis constructed after protracted labor, which is based on a preceding analysis of the sequence of natural numbers as it presents itself, in experience so to speak, to our consideration" [Heijenoort, 1967, 99].

${ }^{13}$ See [Detlefsen, 2012, 208-210] for more on that aspect.

${ }^{14}$ In [Haffner, 2014], I continued the idea of such a synonymy - or maybe an ambiguity - between provable and definable, emphasizing the role played by arithmetic in satisfying Dedekind's principle.
} 
structivists or intuitionnists for whom natural numbers were given by intuition (Poincaré, Brouwer), or by experience (Helmholtz) or by God (Kronecker). It likens him to Leibniz's "formalist" approach, who proved in the New Essays on Human Understanding (book IV, chap. VII, §10) that a statement such as " $2+2$ equals 4", seen as "intuitive" by Descartes, can be proved from the definitions of 1,2, 3 and the properties of equalities, approximately corresponding to the common notion in Euclid's Elements: "Things which equal the same thing also equal one another." [Dedekind, 2008, 96, my translation]

The reference to Leibniz, here, is a way to emphasize that in the applications of the Dedekind principle, what is central is the organization of the conceptual architecture, which provides a framework in which to develop proofs. Providing new definitions based on unprovable premisses is a step towards providing a deductive hierarchy allowing for proofs of all propositions "capable of proof".

\section{$2.2 \quad$ Rigor in the making?}

In the mid-1870s, Dedekind undertook the project of finding a rigorous foundation for module theory. For several years, he conducted researches to "obtain this theory from the smallest number of fundamental laws" [Dedekind, 1897, 113]. However, this time, the outcome was slightly different: rather than a sounder foundation for the theory he was investigating, Dedekind identified the basis for a completely new concept, the Dualgruppe, which is formally equivalent to a lattice. ${ }^{15}$ The genesis of these researches, which lasted almost two decades, can be found in Dedekind's Nachlass, as I mentioned in the introduction.

Studying these manuscripts, one can see that Dedekind's research process goes through several phases. The first phase is close to experimentation, in which Dedekind was exploring the properties of modules and their operations. He used computations and diagrams as ways to experiment, to better understand the laws of module theory by manipulating simple cases, testing on progressively more general cases, trying to identify the laws likely to hold in the general case, and the ones that do not. The second phase was a phase of clarification of the results obtained. The third phase was that of textualization, that is, the writing of a publishable text. But - at

\footnotetext{
${ }^{15}$ [Dedekind, 1897, Dedekind, 1900]. For more on Dualgruppen and the history of lattice theory, one can refer to [Mehrtens, 1979, Corry, 2004, Schlimm, 2011].
} 
least in the case of Dualgruppen - the conceptual development did not follow these steps in a linear way. Indeed, Dedekind wrote several texts which were steps of the conceptual development, and were either aborted or abandoned. These texts were then followed by new phases of experimentation and clarification, and eventually a new (different) text that showed a further step in the development towards the theory of Dualgruppen as exposed in [Dedekind, 1897, Dedekind, 1900].

These manuscripts illustrate a remark, made by Dedekind himself, regarding the difference between the published presentation of the theory and the "historical presentation of the chain of thought" in the case of ideal theory: the way one invents a concept (or finds a result) and the way one choses to define it (or expose it) are not necessarily the same.

Commenting on [Kronecker, 1882], in which Kronecker criticized his definition of ideals, Dedekind stated the following:

It is further said that I "put the concept of all the actual numbers divisible by an ideal divisor at the core of the development" (...) it might seem that I defined the "ideal" through Kummer's "ideal numbers", while I rather give priority to the completely independent invariant definition by the two properties I and $\mathrm{II}^{16}$ (Dirichlet [Vorlesungen über Zahlentheorie] §163 of the second [edition], §167-168 of the third, ${ }^{17}$ also $\S \S 11,19$ in Sur la théorie des nombres entiers algébriques $\left.{ }^{18}\right)$. Maybe this remark of Kronecker's is caused by the historical presentation of the chain of thought which led to the introduction of my concept of ideal (introduction of [Dedekind, 1877a] p. 8-10)! [Edwards et al., 1982, $63]$

Indeed, in [Dedekind, 1877a], Dedekind reminded the reader that Kummer "did not define ideal numbers themselves, but only the divisibility of these numbers" :

If a number $\alpha$ has a certain property $A$, to the effect that $\alpha$ satisfies one or more congruences, he says that $\alpha$ is divisible by an

\footnotetext{
${ }^{16}$ Dedekind means the following definition for an ideal $\mathfrak{a}$ : "I. The sum and difference of any two numbers in the system $\mathfrak{a}$ are always numbers in the same system $\mathfrak{a}$. II. Any product of a number in the system $\mathfrak{a}$ by a number of the system $\mathfrak{o}$ is a number in the system a."

${ }^{17}$ Dedekind refers to his Supplements X and XI in respectively the 1871 and 1879 editions of Vorlesungen über Zahlentheorie, namely [Dedekind, 1871] and [Dedekind, 1879].

18 [Dedekind, 1877a]
} 
ideal number corresponding to the property $A$. [Dedekind, 1877a, $57]$

For Dedekind, this was not a completely satisfying definition because it relied too much on the "analogy with the theory of rational numbers, [which] may lead to hasty conclusions and incomplete proofs" [Dedekind, 1877a, 57]. To solve this, he proposed the well-known alternative:

[I]t will be necessary and sufficient to establish once and for all the common characteristic of the properties $A, B, C, \ldots$ that serve to introduce the ideal numbers, and then to indicate how one can derive, from properties $A, B$ corresponding to particular ideal numbers, the property $C$ corresponding to their product. This problem is essentially simplified by the following considerations. Since a characteristic property $A$ serves to define, not an ideal number itself, but only the divisibility of the numbers in $\mathfrak{o}$ by the ideal number, one is naturally led to consider the set $\mathfrak{a}$ of all numbers $\alpha$ of the domain $\mathfrak{o}$ which are divisible by a particular ideal number. I now call such a system an ideal for short, so that for each particular ideal number there corresponds a particular ideal a. [Dedekind, 1877a, 57-58]

However, this is not the definition of an ideal, it is just an insight into Dedekind's research process at the time. Certainly, concerns with rigor were not foreign to Dedekind's generalization of Kummer's ideal numbers. But after an analysis of the problem at play that led to identifying a solution, it was necessary to go through a second analysis that could give a proper, rigorous definition. Such a definition is given by two necessary and sufficient properties of closure (see footnote 16) which constitute a definition that is "independent" and "invariant" in the sense that it does not depend on the elements of the ideal (and consequently does not depend on the field either).

This is itself an example of how rigor can intervene in the development of mathematical concepts: in a second step in the research process, that of finding the most appropriate definition or proof. The Dedekind principle appears, here, to be essentially applicable to completed researches, so as to organize the results. It is important to note, however, that for Dedekind, rigor was necessary to understand mathematics, as illustrated by the following statement made in a letter to Weber, in 1874, when they started discussing Riemann's works in the context of the edition of his collected works: 
I certainly know [Riemann's] works, and I believe in them, but I do not master them, and I will not master them until having overcome in my way, with the rigor that is customary in number theory, a whole series of obscurities. [Scheel, 2014, 50]

It suggests that rigor played a role in Dedekind's research process, that it was one of his tools for deepening and maybe extending mathematical knowledge, and that it did not solely appear in a step of rigorization close to textualisation. What is, in that case, rigorous mathematics in the making?

Following the road leading to the invention of Dualgruppen, I will show that rigor as the Dedekind principle is not, in fact, present throughout the researches, but that rigor certainly appears as an impetus and a support for mathematical research, albeit in a maybe slightly weaker form.

\section{In Dedekind's workshop: the genesis of a law in Dualgruppe theory}

\subsection{Dualgruppen and their module-theoretic origins}

In [Dedekind, 1871], Dedekind introduced the notion of module, a "system $\mathfrak{a}$ of real or complex numbers $\alpha$ whose sums and differences themselves belong to a" [Dedekind, 1871, 42]. Modules play a central role in Dedekind's number theory. To develop module theory, Dedekind defined:

- a divisibility relation for modules: a module $\mathfrak{a}$ divides a module $\mathfrak{b}$ if $\mathfrak{b}$ is included in $\mathfrak{a}$;

- a notion of "least common multiple" of modules: the LCM of $\mathfrak{a}$ and $\mathfrak{b}$ is the intersection of $\mathfrak{a}$ and $\mathfrak{b}$;

- a notion of "greatest common divisor" of modules: the GCD of $\mathfrak{a}$ and $\mathfrak{b}$ is the module composed by all the numbers $\alpha+\beta$ with $\alpha$ and $\beta$ respectively running through all numbers of $\mathfrak{a}$ and $\mathfrak{b}$.

In [Dedekind, 1877b], Dedekind introduced notations for divisibility, LCMs and GCDs of modules:

- $\mathfrak{a}$ divides $\mathfrak{b}$ is denoted by $\mathfrak{a}<\mathfrak{b}$ or $\mathfrak{b}>\mathfrak{a}$;

- the GCD of $\mathfrak{a}$ and $\mathfrak{b}$ is denoted by $\mathfrak{a}+\mathfrak{b}$;

- their LCM of $\mathfrak{a}$ and $\mathfrak{b}$ is denoted by $\mathfrak{a}-\mathfrak{b}$. 
It allowed him to notice some interesting properties and state new theorems such as, for modules $\mathfrak{a}, \mathfrak{b}, \mathfrak{c}$ with $\mathfrak{a}<\mathfrak{b}$ :

$$
\begin{aligned}
(\mathfrak{a}+\mathfrak{b})-(\mathfrak{a}+\mathfrak{c}) & =\mathfrak{a}+(\mathfrak{b}-(\mathfrak{a}+\mathfrak{c})) \\
(\mathfrak{a}-\mathfrak{b})+(\mathfrak{a}-\mathfrak{c}) & =\mathfrak{a}-(\mathfrak{b}+(\mathfrak{a}-\mathfrak{c}))
\end{aligned}
$$

or again

$$
\mathfrak{a} \pm(\mathfrak{b} \mp \mathfrak{c})=\mathfrak{a} \pm(\mathfrak{b} \mp \mathfrak{c}) .
$$

The last equality corresponds to what are now called the "modular laws" in lattice theory.

Dedekind noted that these "characteristic theorems" for modules display a "peculiar dualism" for the notions of GCD and LCM, that is, any true formula expressed in terms of + and - can be transformed into another true formula by switching these symbols. Dedekind's interest for this dualism led him to investigate further the operations for modules, and eventually to introduce the notion of Dualgruppe. Dedekind presented his Dualgruppe theory in [Dedekind, 1897] and [Dedekind, 1900].

A Dualgruppe is defined in the following manner:

A system $\mathfrak{A}$ of things $\alpha, \beta, \gamma, \ldots$ is called a Dualgruppe, if there are two operations \pm , such that they create from two things $\alpha, \beta$, two things $\alpha \pm \beta$, that are also in $\mathfrak{A}$ and that satisfy [commutativity for + and - , associativity for + and - , and $\alpha \pm(\alpha \mp \beta)=\alpha$ (absorption)]. [Dedekind, 1897, 113], transl. in [Schlimm, 2011].

There exist examples of Dualgruppen in many domains of mathematics. Dedekind gives the following: Schröder's logic, modules, ideals, infinite Abelian groups, Galois groups, fields, number spaces ${ }^{19}$ [Dedekind, 1897, 113114].

Dedekind defined a divisibility relationship, denoted by $\mathfrak{a}<\mathfrak{b}$ or $\mathfrak{b}>\mathfrak{a}$ $(\mathfrak{a}$ a divisor of $\mathfrak{b})$ such that

$$
\mathfrak{a}<\mathfrak{b} \Longleftrightarrow \mathfrak{a}+\mathfrak{b}=\mathfrak{a}, \mathfrak{a}-\mathfrak{b}=\mathfrak{b} .
$$

It verifies the laws of a partial order relation. Note that while Dedekind initially introduced divisibility for modules and then the notions of GCD and

\footnotetext{
${ }^{19}$ Dedekind considers systems such that "each element $a$ is a sequence of $n$ real numbers" taken as coordinates of $a$ such that "each point is a mapping of the system of the $n$ first natural numbers $1,2, \ldots, n$ in the sequence of natural numbers. The points $a \phi b, a \psi b$ must be defined as the $\nu$-coordinate $(a \phi b)_{\nu}$ always the algebraic smallest, and $(a \psi b)_{\nu}$ the greatest of both coordinates $a_{\nu}$ and $b_{\nu} "$ (Cod. Ms. Dedekind XI 1, 4-5).
} 
LCM of modules, he later reversed the order of definition: the order relation (divisibility) is defined according to the operations (GCM and LCM).

The law

$$
(\mathfrak{a}+\mathfrak{b})-(\mathfrak{a}+\mathfrak{c})-(\mathfrak{b}+\mathfrak{c})=(\mathfrak{a}-\mathfrak{b})+(\mathfrak{a}-\mathfrak{c})+(\mathfrak{b}-\mathfrak{c})
$$

is called Idealgesetz, because Dualgruppen formed by ideals (for example, the Dualgruppe generated by three ideals) verify that law. Some Dualgruppen verify what Dedekind called the Modulgesetz (modular law): if $\mathfrak{d}<\mathfrak{m}$, then $(\mathfrak{m}+\mathfrak{p})-\mathfrak{d}=(\mathfrak{p}-\mathfrak{d})+\mathfrak{m}$. The Modulgesetz cannot be derived from the fundamental laws. It should be considered as extending them. Its name comes again from the fact that Dualgruppen formed by modules (for example, the Dualgruppe generated by three modules) verify this law. ${ }^{20}$

Twenty years passed between Dedekind's first and fundamental insight and the publication of articles on the subject. In his Nachlass, one can observe that Dedekind's initial interest in the operations defined between modules led him to explore the possibilities offered by these operations, and by their dualism, with series of examples, tables, diagrams and computations often repeated in slight variations and attempted generalizations. ${ }^{21}$ These computations were gradually more general, and it is through this gradual generalization process that Dedekind was able to isolate the properties related to particular cases from the generally valid ones, to identify which properties should be proven, which properties were to be the fundamental laws (that is, those which cannot be deduced from other laws, such as associativity), and which became core results of the theory. From these computations emerge two main tendencies in Dedekind's research: on one hand, Dedekind shows a deeper interest in the possibility of applying the operations to objects other than modules, and he tries to study them independently from the nature of the operands; on the other hand, his focus slowly shifts from the operations between modules towards what he calls "groups" formed by modules (which are systems closed under the previously defined operations). ${ }^{22}$ These two moves are independent but are eventually

\footnotetext{
${ }^{20}$ Those are respectively the free distributive lattice with three generators, and the free modular lattice with three generators. In [Dedekind, 1897], Dualgruppen verifying the Idealgesetz are called "of the ideal type" (Dualgruppen vom Idealtypus) and those verifying the Modulgesetz are "of the module type" (Dualgruppen vom Modultypus).

${ }^{21}$ For a more detailed analysis of this, see [Haffner, pear].

${ }^{22}$ The term "group", in Dedekind's writings, is used with a relatively large and fluid meaning. Most of the time, Dedekind used the word "group" as he understood it from Galois's works: a set of elements closed under one or two given operations. The properties
} 
merged. Dedekind's Dualgruppe concept is the result of the fusion of these two aspects of his researches: the generalization from modules to any elements satisfying the fundamental laws, and the passage to the level of sets.

\subsection{First elements on Dedekind's research process}

Dedekind's approach in these drafts can be related to what I called the "conceptual analysis", which leads to the identification of the necessary and sufficient conditions for a property to be generally valid. However, as Dedekind is exploring a fairly new domain, his work seems to consist both in the "happy trial and error" process that was mentioned by Jacobi as part of his own research process, and a quasi-systematic research of fundamental laws and proofs that can be linked to Dedekind's ideal of rigor.

Considering the content of these investigations, the question of intuition is not as central as it can be for irrational or natural numbers, or even for algebraic curves. That being said, it is important to note that despite his strong advocacy against geometrical or visual representations in his publications, Dedekind does use such visual aids in his research process. Indeed, some of his drafts display diagrams for the order relation. ${ }^{23}$ Moreover, Dedekind made an extensive use of tables in his drafts (some of which are reproduced in [Dedekind, 1900]). Most of these tables were various ways of presenting the same content (the Dualgruppe generated by three modules), which suggests that Dedekind was looking for the best way to display maybe even visualize - the content and properties of said group, and in particular its duality or symmetry properties. These diagrams and tables can certainly be considered as heuristic tools. For Dedekind, this was perfectly acceptable - as was the use of intuition as a heuristic or pedagogical tool ${ }^{24}$ under the condition that it stayed purely heuristic.

The research process noticeably departs from Dedekind's rule of starting from the most general standpoint. Indeed, in his researches there arises a progressive and stepwise generalization that happens by doing gradually more general computations. Dedekind started by working on modules, and operated on different levels of generality, from numerical cases of finitely generated modules to arbitrary modules. This process is spread through the

of the operations would give defining properties of the "group" in question - properties such as associativity were not, for example, included in his general idea of a "group". It is the use made in his drafts on Dualgruppen. In this paper, I will use the term "group" as an actor's category and follow Dedekind's use of the word.

${ }^{23}$ See [Haffner, 2019].

${ }^{24}$ [Dedekind, 1872, 767] 
many years of research that can be witnessed in Dedekind's drafts. It appears that computations and their generalizations are not a stage in the research process, but developed in parallel and in relation to the reflections leading to the definition of the concept of Dualgruppe, as well as the identification of interesting properties or theorems. In fact, this process of experimentations and step-wise generalizations gives Dedekind key elements to observe the properties and laws verified by the operations. From this viewpoint, his approach resembles an inductive one. The trial-and-error and systematic explorations of the properties of modules and their operations might be a way to examine his intuitions about module-theoretical properties, to verify and prove them - and thus eliminate intuition.

In the course of his investigation, in endeavours to identify the fundamental properties of the operations, Dedekind tried to detach the operations from their interpretation in module theory. Hence, Dedekind started by giving himself various questions to solve so as to study the properties of the operations (and the group formed) for objects such as groups (Abelian, nonAbelian, Hamiltonian, non-Hamiltonian, Galois, ... ), ideals or "elements". Through the resolution of these self-assigned problems, he gradually increased the generality of the operations he worked with, and progressed towards a greater grasp of their properties. He thus advanced towards studying the operations not as GCD and LCM of modules, but as indeterminate binary operations, a way to combine elements whose individual nature is also left indeterminate. Hence, the research was taken away from modules themselves, and the interest was going to the operations considered independently of the nature of the operands.

Eventually, Dedekind's Dualgruppe theory does hold to his standard of generality, that is, having definitions and laws covering all possible cases at once. But this is achieved through a process that appears to be more inductive than deductive. As a matter of fact, the appropriate deductive hierarchy only slowly emerges as a result of Dedekind's systematic experimentations. It goes through several phases of verification, in which Dedekind tests and sometimes modifies the order of exposition of the properties. This suggests that this part of his ideal of rigor might be related to a posteriori verifications or justifications, and to the process of textualisation, rather than to the mathematical research.

In the next paragraph, I give an example of the gradual clarification of the status of a given property, named Modulgeset ${ }^{25}$ by Dedekind, and of

${ }^{25}$ The law is named fairly late in the process, in the next-to-last draft of [Dedekind, 1900]. However, for readability purposes, I will refer to the law as Modulgesetz. 
how its place in the deductive hierarchy evolved throughout the researches.

\subsection{Gradual clarification of the properties: the example of the Modulgesetz}

It is through this process of explorations and gradual clarifications that the importance and specificity of the "modular law" (Modulgesetz) are put forward. This law can be stated as follow: for three modules $\mathfrak{p}, \mathfrak{m}, \mathfrak{d}$, with $\mathfrak{d}<\mathfrak{m}$, we have

$$
(\mathfrak{p}+\mathfrak{m})-\mathfrak{d}=(\mathfrak{p}-\mathfrak{d})+\mathfrak{m}
$$

or, with $\mathfrak{p}=\mathfrak{a}, \mathfrak{m}=\mathfrak{b}-\mathfrak{c}$ and $\mathfrak{d}=\mathfrak{a}+\mathfrak{b}$

$$
(\mathfrak{a}+(\mathfrak{b}-\mathfrak{c}))-(\mathfrak{b}+\mathfrak{c})=(\mathfrak{a}-(\mathfrak{b}+\mathfrak{c}))+(\mathfrak{b}-\mathfrak{c}) .
$$

The law first appeared in published works in [Dedekind, 1894, 499] as a property of modules. In the earliest drafts (between 1871 and the 1880s) and in [Dedekind, 1877b], the following properties are given:

$$
\begin{aligned}
& (\mathfrak{a}+\mathfrak{b})-(\mathfrak{a}+\mathfrak{c})=\mathfrak{a}+(\mathfrak{b}-(\mathfrak{a}+\mathfrak{c})) \\
& (\mathfrak{a}-\mathfrak{b})+(\mathfrak{a}-\mathfrak{c})=\mathfrak{a}-(\mathfrak{b}+(\mathfrak{a}-\mathfrak{c}))
\end{aligned}
$$

But the Modulgesetz is absent. In later drafts, it starts to appear and to be used in computations (in particular, when Dedekind wants to compute all the elements of a given "group of modules"). It seems that these uses of the Modulgesetz prompted Dedekind to investigate its conditions of validity in the late 1880s. His main concern was to determine in which conditions the law could be generally valid - but still only for modules.

In the following, I will give some examples of the path followed. While the choice of the excerpts from Dedekind's Nachlass remains a relatively subjective one, I did my best to choose representative folios, taking into account that Dedekind, in these drafts, repeats himself fairly often.

In a couple of sheets from the period between 1885 and the early 1890 s, Dedekind considered three finitely generated modules $\mathfrak{a}, \mathfrak{b}, \mathfrak{c}$ with a basis composed of two numbers. These modules, denoted by $[a, b]$ with $a$ and $b$ the real numbers forming the basis, are all the numbers of the form $a x+$ by. It is likely that, in the draft commented on below, the modules are of the form $\mathfrak{a}=\left[h a_{1}, a_{2}+b c \omega\right], \mathfrak{b}=\left[h b_{1}, b_{2}+a c \omega\right], \mathfrak{c}=\left[h c_{1}, c_{2}+a b \omega\right](\omega$ irrational number). For these modules, Dedekind identifies the necessary and

sufficient condition for the validity of the Modulgesetz (Cod. Ms. Dedekind 
$\mathrm{X} 10, \mathrm{p} 5$ and p. $43^{26}$ ). After small computations on GCD and LCM of modules, which mostly aim at understanding conditions for equalities such as $\mathfrak{a}-(\mathfrak{b}+\mathfrak{c})=\mathfrak{a}-\mathfrak{c}$, Dedekind asks:

When is

$$
(\mathfrak{a}+\mathfrak{b})-\mathfrak{c}=(\mathfrak{a}-\mathfrak{c})+\mathfrak{b}
$$

general? (Cod. Ms. Dedekind X 10, p. 43)

Using his previous computations for this special case, he identifies the appropriate values for the coefficients of the basis of the modules involved. Putting back the coefficients' values into the modules', Dedekind is able to pin down the necessary property of the initial modules for the Modulgesetz to be valid, that is:

$$
\mathfrak{b}>\mathfrak{c} .
$$

In a second page (Cod. Ms. Dedekind X 10, p. 5), Dedekind briefly shows that $\mathfrak{b}>\mathfrak{c}$ is also a sufficient condition for the validity of the Modulgesetz in this specific case.

These notes go together with many others on finitely generated modules. From there, we find many small notes on the Modulgesetz and how to prove it, with different levels of generality, sometimes on the side of the page for other works on modules written down with a different pen. It is likely that the importance of the so-called Modulgesetz was brought out in the course of these researches on modules (and in particular on the group generated by three modules). This very experimental approach seems rather removed from the Dedekindian ideal of rigor. It is interesting to note how Dedekind worked his way towards a general proof: he adopted a very case-by-case approach before actually attempting a general (that is, for any three modules, or even for any elements $a, b, c$ verifying the laws of + and - ) proof.

It became a recurring concern of Dedekind's around 1890 to prove that for three arbitrary modules $\mathfrak{a}, \mathfrak{b}, \mathfrak{c}$ if $\mathfrak{b}>\mathfrak{c}$, then $(\mathfrak{a}+\mathfrak{b})-\mathfrak{c}=(\mathfrak{a}-\mathfrak{c})+\mathfrak{b}$, using only the axioms for + and - and the definition of divisibility - or, in Dedekind's own words, to prove it "without new principles". At least a dozen sheets consider this question, all interrupted or deemed "insufficient" (ungenügend) by Dedekind. In this sense, it is a clear illustration of the Dedekind principle in action. However, Dedekind slowly came to realise

\footnotetext{
${ }^{26}$ Even though these sheets are not together in the file, their content and format strongly suggest that they were written together. As explained at the beginning of the archive's catalog, Dedekind's Nachlass was donated to the Göttingen archive by Dedekind's heirs in 1931 and catalogued as such. Many of the files appear to be very fragmentary or even messy.
} 
that this proof was an impossible task.

In (Cod. Ms. Dedekind X 11-1, p. 12), Dedekind starts by listing all the possible versions of (i), such as: ${ }^{27}$

$$
\begin{aligned}
(\mathfrak{a}+\mathfrak{b})-(\mathfrak{a}+\mathfrak{c}) & =\mathfrak{a}+(\mathfrak{b}-(\mathfrak{a}+\mathfrak{c}))=\mathfrak{a}+(\mathfrak{c}-(\mathfrak{a}+\mathfrak{b})) \\
(\mathfrak{b}+\mathfrak{c})-(\mathfrak{a}+\mathfrak{b}) & =\mathfrak{b}+(\mathfrak{c}-(\mathfrak{a}+\mathfrak{b}))=\mathfrak{b}+(\mathfrak{a}-(\mathfrak{b}+\mathfrak{c})) \\
(\mathfrak{c}+\mathfrak{a})-(\mathfrak{b}+\mathfrak{c}) & =\mathfrak{c}+(\mathfrak{a}-(\mathfrak{b}+\mathfrak{c}))=\mathfrak{c}+(\mathfrak{b}-(\mathfrak{c}+\mathfrak{a})) \\
(\mathfrak{a}-\mathfrak{b})+(\mathfrak{a}-\mathfrak{c}) & =\mathfrak{a}-(\mathfrak{b}+(\mathfrak{a}-\mathfrak{c}))=\mathfrak{a}-(\mathfrak{c}+(\mathfrak{a}-\mathfrak{b})) \\
(\mathfrak{b}-\mathfrak{c})+(\mathfrak{a}-\mathfrak{b})=\mathfrak{b}-(\mathfrak{c}+(\mathfrak{a}-\mathfrak{b})) & =\mathfrak{b}-(\mathfrak{a}+(\mathfrak{b}-\mathfrak{c}))
\end{aligned}
$$

He then considers various cases: when $\mathfrak{c}=\mathfrak{a}$, when $\mathfrak{c}=\mathfrak{a}+\mathfrak{b}$, when $\mathfrak{c}=\mathfrak{a}-\mathfrak{b}$, and looks at how each case modifies the equalities in question. For example, for $\mathfrak{c}=\mathfrak{a}$, it gives us:

$$
\begin{aligned}
(\mathfrak{a}+\mathfrak{b})-\mathfrak{a}=\mathfrak{a}+(\mathfrak{a}-\mathfrak{b}) & =\mathfrak{a}+\{(\mathfrak{a}+\mathfrak{b})-\mathfrak{a}\} \\
\mathfrak{a}+\mathfrak{b} & =\mathfrak{b}+\{(\mathfrak{a}+\mathfrak{b})-\mathfrak{a}\} \\
\mathfrak{a}+(\mathfrak{a}-\mathfrak{b})=\mathfrak{a}-(\mathfrak{a}+\mathfrak{b}) & =\mathfrak{a}-\{\mathfrak{a}+(\mathfrak{a}-\mathfrak{b})\} \\
\mathfrak{a}-\mathfrak{b}=\mathfrak{b}-(\mathfrak{c}+(\mathfrak{a}-\mathfrak{b})) & =\mathfrak{b}-\{\mathfrak{a}+(\mathfrak{a}-\mathfrak{b})\}
\end{aligned}
$$

He shows that all of this is equivalent with:

$$
\mathfrak{a}=\mathfrak{a}+(\mathfrak{a}-\mathfrak{b}) ; \mathfrak{a}=\mathfrak{a}-(\mathfrak{a}+\mathfrak{b})
$$

that is, the absorption law (which is a property that will later be added to the fundamental properties of the operations in place of idempotence, as we will see below). Following this, Dedekind writes:

Theorem: If $\mathfrak{m}>\mathfrak{d}$, that is

$$
\mathfrak{m}+\mathfrak{d}=\mathfrak{d} ; \mathfrak{m}-\mathfrak{d}=\mathfrak{m}
$$

and $\mathfrak{p}$ arbitrary, then we have

$$
(\mathfrak{p}+\mathfrak{m})-\mathfrak{d}=(\mathfrak{p}-\mathfrak{d})+\mathfrak{m}
$$

[In the margin:] other writings:

$$
(\mathfrak{m}+\mathfrak{p})-\mathfrak{d}=\mathfrak{m}+(\mathfrak{p}-\mathfrak{d})
$$

\footnotetext{
${ }^{27}$ Here and below, the numbering of the equations is Dedekind's.
} 
or

$$
\mathfrak{d}-(\mathfrak{p}+\mathfrak{m})=(\mathfrak{d}-\mathfrak{p})+\mathfrak{m}
$$

[Back to main text:] Proof (Attempt without new principles).

One sets

$$
\mathfrak{a}=(\mathfrak{p}+\mathfrak{m})-\mathfrak{d} ; \mathfrak{b}=(\mathfrak{p}-\mathfrak{d})+\mathfrak{m}
$$

We have 28

$$
\begin{aligned}
& \mathfrak{b}+\mathfrak{p}=\mathfrak{p}+(\mathfrak{p}-\mathfrak{d})+\mathfrak{m}=\mathfrak{p}+\mathfrak{m} \quad \mathfrak{b}>\mathfrak{p}+\mathfrak{m} \\
& \mathfrak{b}+\mathfrak{m}=\mathfrak{b} \quad\} \quad \mathfrak{m}>\mathfrak{b} \\
& \mathfrak{b}+\mathfrak{d}=\mathfrak{d}+(\mathfrak{p}-\mathfrak{d})+\mathfrak{m}=\mathfrak{d}+\mathfrak{m}=\mathfrak{d}\} \quad \mathfrak{b}>\mathfrak{d} \\
& \mathfrak{a}-\mathfrak{p}=\mathfrak{p}-\mathfrak{d} ; \quad \mathfrak{p}-\mathfrak{d}>\mathfrak{a} \\
& \left.\begin{array}{l}
\mathfrak{b}+\mathfrak{m}=\mathfrak{b} \\
\mathfrak{b}+\mathfrak{d}=\mathfrak{d}+(\mathfrak{p}-\mathfrak{d})+\mathfrak{m}=\mathfrak{d}+\mathfrak{m}=\mathfrak{d}
\end{array}\right\} \quad \mathfrak{m}>\mathfrak{a}>\mathfrak{d}
\end{aligned}
$$

All of this only tells us that $\mathfrak{b}>\mathfrak{a}$.

$$
\mathfrak{a}=(\mathfrak{b}+\mathfrak{p})-\mathfrak{d} ; \mathfrak{b}=(\mathfrak{a}-\mathfrak{p})+\mathfrak{m a}>\mathfrak{b}+\mathfrak{p} ; \mathfrak{b}<\mathfrak{a}-\mathfrak{p}
$$

The proof that $\mathfrak{a}<\mathfrak{b}$ does not succeed (with the above principles alone).

Note that Dedekind focuses on proving the laws from the fundamental properties alone, thus basing his (attempt at a) proof on unprovable premisses, and avoiding the use of external tools of results.

The impossibility of such a proof is Dedekind's eventual conclusion, in [Dedekind, 1897, Dedekind, 1900]:

But this Modulgesetz is, as I showed in the $§ 4$ of my [[Dedekind, 1897]], unfortunately not derivable from the fundamental laws [commutativity, associativity and absorption for + and -] and constitutes therefore an essential extension of them for module theory. [Dedekind, 1900, 239, Dedekind's emphasis]

The result he alludes to, in [Dedekind, 1897], is the proof that there exist $D u$ algruppen which do not verify the modular law. But this was not Dedekind's immediate conclusion, likely because upon arriving at the observation that the Modulgesetz was not a consequence of the laws of the operations and divisibility, Dedekind still hadn't introduced the concept of Dualgruppe, nor had he extended his investigations to other objects.

\footnotetext{
${ }^{28}$ The absorption law is what allows Dedekind to draw these equalities
} 
At this point, the exploration conducted by Dedekind is very systematic. Dedekind goes through every possibility to understand and prove the status of the Modulgesetz, to eliminate any superfluous assumptions. Dedekind went on to investigate the Modulgesetz's status from another viewpoint: he tried to understand what happened in situations in which the law does not hold. His first attempts at studying cases without the modular law were inconclusive. For example, in (Cod. Ms. Dedekind X 11-1 p. 15), he takes the following initial condition

$$
(\mathfrak{a}+\mathfrak{b})-\mathfrak{c}<\mathfrak{a}+(\mathfrak{b}-(\mathfrak{c}+\mathfrak{a}))
$$

which renders the Modulgesetz invalid. Taking a numerical example, he observed that he could not generate a group as he did in other cases. This led him to conclude, in a rare occurence of a dated result:

17 November 1890. This example of an additive group shows the indispensability of the law (which does not hold here)

$$
(p+m)-d=(p-d)+m
$$

$m$ divisible by $d$, that is, $m+d=d$. (Cod. Ms. Dedekind X 11-1 p. 15)

The idea that the Modulgesetz could be "indispensable" shows that Dedekind had not broadened his investigations outside of module theory. In anachronistic terms, what Dedekind seemed to identify here is the defining role of the Modulgesetz for modular lattices. But, at this point he did not have the general definition of Dualgruppen, and likely hadn't tried to generalize these researches: he was still investigating the properties of the operations defined for modules, and in particular their property of duality.

Hence, for some time, he considered that the Modulgesetz was an "indispensable" law - without considering it a defining law (or axiom). He even considered it the "source of duality" for the theory of modules. In (Cod. Ms. Dedekind X 11-1, pp. 13-14), which can be dated from the early to mid-1890s, Dedekind studied the divisibility laws for three modules $\mathfrak{m}, \mathfrak{d}$, $\mathfrak{p}$ with $\mathfrak{d}$ divides $\mathfrak{m}$. The first two pages are covered with computations for the divisibility of the three modules (with all possible additions), tables of GCDs, LCMs, multiples, and the systematic verification of properties such as associativity. These considerations end with a short paragraph, which seems to conclude the previous computations, which draws on (the absence of) links between the defining laws of addition and the Modulgesetz: 
The source of dualism in the theory of modules, that is, the following theorem:

I. If $\mathfrak{m}$ is divisible by $\mathfrak{d}$, then $(\mathfrak{m}+\mathfrak{p})-\mathfrak{d}=\mathfrak{m}+(\mathfrak{p}-\mathfrak{d})$ amounts to what follows (for the sign + ):

II. If $\mathfrak{m}+\mathfrak{d}=\mathfrak{d}, \mathfrak{a}+\mathfrak{m}+\mathfrak{p}=\mathfrak{m}+\mathfrak{p}, \mathfrak{a}+\mathfrak{d}=\mathfrak{d}$ then there exists at least one element (module) $\mathfrak{q}$ verifying the conditions

$$
\mathfrak{q}+\mathfrak{p}=\mathfrak{p}, \mathfrak{q}+\mathfrak{d}=\mathfrak{d}, \mathfrak{a}+\mathfrak{m}+\mathfrak{q}=\mathfrak{m}+\mathfrak{q}
$$

Or again (if one sets $\mathfrak{d}=\mathfrak{m}+\mathfrak{p}^{\prime}$ )

III. If $\mathfrak{a}+\mathfrak{m}+\mathfrak{p}=\mathfrak{m}+\mathfrak{p}, \mathfrak{a}+\mathfrak{m}+\mathfrak{p}^{\prime}=\mathfrak{m}+\mathfrak{p}^{\prime}$, then we have at least one element $\mathfrak{q}$ verifying the conditions:

$$
\mathfrak{q}+\mathfrak{p}=\mathfrak{p}, \mathfrak{q}+\mathfrak{m}+\mathfrak{p}^{\prime}=\mathfrak{m}+\mathfrak{p}^{\prime}, \mathfrak{a}+\mathfrak{m}+\mathfrak{q}=\mathfrak{m}+\mathfrak{q} .
$$

But this theorem (I or II or III) is not in any way a necessary consequence of the three laws of pure addition:

IV. $\mathfrak{a}+\mathfrak{a}=\mathfrak{a}, \mathfrak{a}+\mathfrak{b}=\mathfrak{b}+\mathfrak{a},(\mathfrak{a}+\mathfrak{b})+\mathfrak{c}=\mathfrak{a}+(\mathfrak{b}+\mathfrak{c})$.

(Cod. Ms. Dedekind X 11-1, p 14v)

The Modulgesetz's status as the "source of dualism" is short-lived. Yet, it is a significant moment in the genesis of the Dualgruppe concept (which, at this point, still has a long way to come). As I mentioned, this law is central for studying the group generated by three modules (that is, the free modular lattice with three generators) which is, in the early 1890s, Dedekind's main interest.

What Dedekind probably hadn't seen, here, is that this duality is not a property of modules (or, to say it with an anachronism, it is not a property of modular lattices) and that he could define more general operations verifying the same properties as the operations for modules (that is, anachronistically again, that he could define a general concept of lattice). This changed with the application of the operations to more diverse objects, and later on with their definition in a more general setting (in what Dedekind called a "logical theory"). ${ }^{29}$ Only then does it become clear that the law presented as the "source of dualism" is a property of modules (which gave it its name), that is, related to the restricted context in which he was initially working;

\footnotetext{
${ }^{29}$ In Dedekind's Nachlass, there are several sets of notes on Schröder's Algebra der Logik. Although the datation is difficult, it is possible (if not likely) that learning of Schröder's logical calculus - which is consistently cited by Dedekind as an instance of Dualgruppen - played a significant role in his reflection.
} 
that duality is neither dependent nor related to it; and that there exist nonmodular Dualgruppen. These three conclusions are entangled and certainly did not follow a linear process. They are related to the status of the Modulgesetz.

An important step towards the general concept of Dualgruppe is what Dedekind called the "generalisation of a part of module theory" in (Cod. Ms. Dedekind X 11-2, pp. 56-57), which was likely written in the mid1890s. ${ }^{30}$ Dedekind studies here the conditions of possibility for the definition of a group defined by two operations $\phi, \psi$ verifying the same laws as his module-theoretic + and - , within a general framework, and in particular not verifying the (still to be named) Modulgesetz.

Dedekind's first move in this generalization is to change the notations so as to better fit the context in which he will be working. Indeed, + and - are related to the arithmetical study of module theory, introduced in numbertheoretical works, and follow Dedekind's initial desire to mimic arithmetic in module (and ideal) theory. Whereas $\varphi$ and $\psi$ are usually used by Dedekind for 'set' theory, which he calls "logic", as in [Dedekind, 1888] to which he explicitly refers in his drafts. These "logical" notations are thus explicitly introduced here as a step in the development of a "more general theory". ${ }^{31}$ Dedekind considers a system of elements $a, b, \ldots$ and defines two operations $\phi$ and $\psi$ in the following way:

Operation $\phi$ with the laws (addition of modules; gr[eatest] com[mon] divisor)

$$
a \phi a=a ; a \phi b=b \phi a ;(a \phi b) \phi c=a \phi(b \phi c)=a \phi b \phi c=\Phi(a, b, c) .
$$

Operation $\psi$ with the laws (sm[allest] com[mon] mult[iple])

$$
a \psi a=a ; a \psi b=b \psi a ;(a \psi b) \psi c=a \psi(b \psi c)=\Psi(a, b, c)=a \psi b \psi c .
$$

Connection between $\phi$ and $\psi$

$$
\text { (III) }(a \phi b) \psi a=a ;(a \psi b) \phi a=a .
$$

\footnotetext{
${ }^{30}$ In particular, the use of the idempotence as a fundamental defining law of the operations tells us that it was written before the text "On the dualism in module theory", presented below, in which Dedekind gives a first fully textualized general presentation of his theory.

${ }^{31}$ However, in the published papers, Dedekind uses + and - .
} 
We have, here, the operations defined by their laws solely, without taking into account the nature of the operands. Having defined the order relation and shown its elementary properties (e.g., transitivity), he shows that it is indeed possible to form a group that only verifies the inequality $m+(a-d)>$ $(m+a)-d$ (with $m>d$ ) and only this inequality - which is different from completely invalidating the Modulgesetz. On the next page, Dedekind identifies "the simplest example in which $m>d$ but $m \phi(a \psi d)$ and $(m \phi a) \psi d$ are different", thus showing that it is indeed possible to form such a group not verifying the Modulgesetz.

The Modulgesetz's status thus changes again, here, and so does its place in the conceptual architecture gradually built by Dedekind. At that point, Dedekind understood that the property is not related to the operations, but to the context in which they were initially defined. It is not an "indispensable" law for the study of the operations (and of the groups thus formed).

The quote from [Dedekind, 1900] given p. 21 shows that when Dedekind clarified and established a general definition for the Dualgruppe concept, he identified that he just had to add the law to the definition to amend the initial definition and circumscribe a particular type of Dualgruppe. This, however, only happened after a period during which the status of the Modulgesetz was that of a law requiring module theory or specific to module theory.

Between 1894 and 1897, Dedekind wrote a text that is best described as a first move towards Dualgruppe theory. The text in question was initially entitled On the dualism in module theory (Cod. Ms. Dedekind XI 1, 1-27), which Dedekind later corrected to be On Dualgruppen. Despite its initial title, this text seems to try to detach the investigation from the specific nature of modules and adopt a more general approach (similar to the researches described above). He starts by defining an unnamed system verifying the following conditions:

I have often noticed a curious dualism, which appears in the theory of modules. ${ }^{32}$ The same is repeated so frequently in other domains of research, that it seems beneficial to understand the general laws of combinations [Verknüpfungs-Gesetze] which prevail in this theory independently of the initial premises on which it is grounded. If such is the case, and that we replace the signs + and - used to designate the greatest common divisor and the least common multiple of two modules, by $\varphi, \psi$, then only the

\footnotetext{
${ }^{32}$ Dirichlet's Vorlesungen über Zahlentheorie, fourth edition, §169. [Dedekind's footnote]
} 
following hypotheses remain:

In a finite or infinite system $S$ of elements $a, b, c, \ldots$ whose meaning is left completely indeterminate, there are two kinds of combinations $\varphi$ and $\psi$, which from any two identical or distinct elements $a, b$ always produce two completely determined elements of the same system $S$ designated by $a \varphi b, a \psi b$. They obey the following six laws

$$
\begin{array}{cc}
a \varphi c=a & (1) \\
a \varphi b=b \varphi a & (2)(1) \\
(a \varphi b) \varphi c=a \varphi(b \varphi c) & (3)(2) \\
a \varphi a=a & \left(1^{\prime}\right) \\
a \psi b=b \psi a & \left(2^{\prime}\right)\left(1^{\prime}\right) \\
(a \psi b) \psi c=a \psi(b \psi c) & \left(3^{\prime}\right)\left(2^{\prime}\right) \\
a \psi(a \varphi b)(a \varphi b) \psi a=a & (4)(3) \\
a \varphi(a \psi b)(a \psi b) \varphi a=a & \left(4^{\prime}\right)\left(3^{\prime}\right) .
\end{array}
$$

(Cod. Ms. Dedekind XI 1, 1-2)

On a side note, let me mention that these striken out laws show us the exact moment when Dedekind realized - as he explained in a note on the back of the page - that the idempotency could be deduced from the absorption law and was, thus, not a fundamental property. ${ }^{33}$ It is another good example of the importance of identifying fundamental propositions for definitions, which means that a definitional property should be (as) unprovable (as possible). It also shows that identifying said fundamental properties can happen fairly late in the research process, and as a matter of fact as part of the textualisation process.

Module theory barely appears in On the dualism in module theory, except in three types of occasions: as an example (Beispiel) of the general theory exposed, in reference to Dedekind's earlier works (for example, when defining the order/divisibility relation), and when the Modulgesetz has to be proved in the third paragraph:

\footnotetext{
33"The last two laws (3) and (3') (...) contain a connection between the operations $\varphi$, $\psi$ and their combination leads - even without reference to the earlier laws - to the two conclusions

$$
\begin{array}{r}
c c a \varphi a=a \\
a \psi a=a
\end{array}
$$

if one replaces the arbitrary element $b$ in $\left(3^{\prime}\right)$ with $a \varphi b$, in (3) with $a \psi b$." (Cod. Ms. Dedekind XI 1, 3v)
} 
We want now to concern ourselves more closely with the theorem (16) $[m \phi(a \psi d)>(m \phi a) \psi d]$. In the module theory (and as well in the three domains of application mentioned in $\$ 1$ [groups, systems of undetermined elements, points of a "space of real numbers of dimension $n "])$, it is possible to prove that from

$$
(m \varphi a) \psi d>m \varphi(a \psi d)
$$

from which, with respect to the theorem III, it follows that

$$
m \varphi(a \psi d)=(m \varphi a) \psi d
$$

For this proof I expressly emphasized ${ }^{34}$ that the previous theorems, which are all based on the six fundamental laws stated in $\S 1$, do not suffice, and that it is rather required to go back, once again, to the concept of module. (Cod. Ms. Dedekind XI 1, p. 10r)

So, here the Modulgesetz is a general law better proved using module theory. It is not the same to simply state that this law is verified by modules, and to state that to prove this law one should "go back to" module theory, as he does here. ${ }^{35}$ To prove a general law using a less general theory seems to go against Dedekind's ideal of rigor. At the same time, his insistance on providing a proof seems to fit in the said ideal of rigor. What seems to be lacking, here, is the appropriate conceptual and deductive hierarchy. Dedekind considered, at this point, that using module theory in the theory of Dualgruppen would give him the Modulgesetz, while, in the final version of the theory, he saw that the Modulgesetz was an additional property of certain types of Dualgruppen (among which, the Dualgruppen formed by modules) under which his previous researches on module theory largely fell.

In On the dualism in module theory, Dualgruppen - which were not yet named - were separated from researches on modules and groups of modules (which Dedekind called Modulgrupen). Dedekind then realized that he needed to merge his researches on Modulgruppen and his general "logical" investigations, as he indicates in a margin note, linking that text to a shorter manuscript entitled Some propositions on Modul-Gruppen (Cod. Ms. Dedekind XI 1, 29-32). This move is crucial for the genesis of Dualgruppen:

\footnotetext{
${ }^{34}$ Dirichlet's Vorlesungen über Zahlentheorie, fourth edition, §169, p. 499 [Dedekind's footnote.]

${ }^{35}$ This imprecision is eventually lifted in later researches on the "general theory".
} 
putting together a very general theory based on the duality of operations defined between two elements, and his investigations into the theory of modules and groups formed by modules.

Before introducing the concept of Dualgruppe, however, Dedekind explored further the possibilities of "generaliz[ing] a part of module theory" and developing a "logical theory". Here, the Modulgesetz is seen as a property of module theory and slowly becomes used to circumscribe "modulelike" parts of the "logical theory". In (Cod. Ms. Dedekind X 11-2, pp. 53, 54, 59 and (probably) 60), we find several large sheets, which were written after the above studied On the dualism in module theory ${ }^{36}$ but still before 1897. Here is a glimpse at what Dedekind was doing. In (Cod. Ms. Dedekind $\mathrm{X}-11-2$, p. 54), he is working with three modules $\mathfrak{a}, \mathfrak{b}, \mathfrak{c}$, and he imposes conditions of divisibility such that the group generated only has eight elements. He then states the following:

There are thus only eight modules left, that is, $\mathfrak{a}, \mathfrak{b}, \mathfrak{c}$ and

$$
\left.\left.\begin{array}{l}
\mathfrak{b}^{\prime \prime \prime}=\mathfrak{a}+\mathfrak{c} \quad ; \mathfrak{c}_{3}=\overline{\mathfrak{a}-\mathfrak{b}} \\
\mathfrak{c}^{\prime \prime \prime}=\mathfrak{a}+\mathfrak{b} \quad ; \mathfrak{b}_{3}=\mathfrak{a}-\mathfrak{c}
\end{array}\right\} \text { and } \mathfrak{d}^{\prime}=\mathfrak{d}_{1}=\begin{array}{c}
(\mathfrak{a}+\mathfrak{b})-\mathfrak{c}=(\mathfrak{a}-\mathfrak{c})+\mathfrak{b} \\
(\mathfrak{b}+\mathfrak{a})-\mathfrak{c}=\mathfrak{b}+(\mathfrak{a}-\mathfrak{c})
\end{array}\right\} \text { in the module theory. }
$$

Here, the Modulgesetz is clearly identified as a property of module theory. Dedekind goes on to the first step of a generalization:

$$
\begin{aligned}
& \text { One replaces } \left.+, \quad-, \quad a, b, \quad c, \quad b^{\prime \prime \prime}, \quad c^{\prime \prime \prime}, \quad b_{3}, \quad c_{3}, \quad d^{\prime}=d_{1}\right\} \text { NB! Consequence of the } \\
& \text { by } \left.\varphi, \quad \psi, \quad a, m, \quad d, d^{\prime \prime \prime}, \quad d^{\prime}, m^{\prime}, m^{\prime \prime \prime}, \underline{d^{\prime \prime}=m^{\prime \prime}}\right\} \text { special module theory }
\end{aligned}
$$

The "Nota Bene" refers only to the underlined property which is a consequence of the Modulgesetz.

The title of the following investigations, starting immediately below the passage just quoted, is indeed "More general (logical) theory". The operations are introduced as indeterminate binary operations, a way to combine elements whose individual nature is also left indeterminate.

General (logical) theory.

Three elements $a, b, c$; laws (general)

(1) $a \varphi b=b \varphi a ;\left(1^{\prime}\right) a \psi b=b \psi a \quad$ from which (when $b$ in $\left(3^{\prime}\right)$ is replaced by

(2) $\left.(a \varphi b) \varphi c=a \varphi(b \varphi c) ;\left(2^{\prime}\right)(a \psi b) \psi c=a \psi(b \psi c)\right\} a \varphi b$, and in (3) by $\left.a \psi b\right)$

(3) $a \psi(a \varphi b)=a ;\left(3^{\prime}\right) a \varphi(a \psi b)=a$

(4) $a \varphi a=a ;\left(4^{\prime}\right) a \psi a=a$.

\footnotetext{
${ }^{36}$ Indeed, the absorption law is given as a fundamental law, following Dedekind's realization that idempotence could be deduced from it.
} 
Dedekind then continues to unfold the laws and compute the possible combinations - which will form the elements of the group generated by the three elements $a, b, c$. He works in a very systematic, almost combinatorial way. Here, the researches are thus completely taken away from modules themselves, and Dedekind's interest is moving towards the operations considered independently from the nature of the operands. The Modulgesetz consequently takes a lesser importance. It appears a few times as circumscribing a "Modul-Art" (modular, or more literally, of the module kind) part of the theory, foreshadowing its later status.

After having introduced a general definition for the concept of Dualgruppe, Dedekind engaged in the study of many special cases of Dualgruppen, such as:

- Numerical cases for the Dualgruppe generated by three modules;

- Dualgruppen without Modulgesetz; Dualgruppe without Modulgesetz and with additional conditions (for example, generated by three elements verifying divisibility conditions);

- Dualgruppen with Modulgesetz; Dualgruppe with Modulgesetz and with additional conditions (for example, generated by three elements verifying divisibility conditions);

- Dualgruppen generated by three ideals; Dualgruppe verifying the Idealgesetz;

- Dualgruppen with Modulgesetz but without Idealgesetz;

- Dualgruppen generated by three Abelian or Hamiltonian groups:

- General Dualgruppen.

At this stage, dating and ordering documents becomes a complicated task, as the concepts, notations, and results stabilize. Some documents that can be dated with certainty from after 1900 (for example, written on the back of a dated invoice) contain researches similar to the ones listed here. Dedekind's interest, thus, still lies in studying various cases of Dualgruppen after 1900 - which shouldn't be surprising since he considered that the many examples of Dualgruppen that could be found were a crucial property of his concept highlighting "how diverse these domains are" [Dedekind, 1897, 113].

The use of systematic case studies seems to allow Dedekind to better distinguish the status of certain properties and their relationships to the 
fundamental laws. In particular, he shows that Dualgruppen verifying the Idealgesetz necessarily verify the Modulgesetz, and finds the simplest examples of a Dualgruppe that does not verify the Modulgesetz and of a Dualgruppe verifying the Modulgesetz but not the Idealgesetz. These results were published in [Dedekind, 1897, 116 sqq.] It is likely that it was by exploring these various possibilities and by combining the conditions in this new conceptual framework that is the Dualgruppe theory, that the stabilization of the properties happened - which, of course, required rigorous proofs afterwards.

The Modulgesetz and its ideal counterpart, the Idealgeset $z^{37}$, are named in [Dedekind, 1897]. Following the presentation of several examples of $D u$ algruppen, he states

But there is a third example, the Dualgruppe consisting of the ideals of a finite[ly generated] field. On these grounds, I will name the dual laws $[(a \pm b) \mp(a \pm c)=a \pm(b \mp c)]$ Idealgesetz, and any Dualgruppe verifying these laws, I want to name Dualgruppe of the ideal type. (...) These laws [several dual versions of the Modulgesetz] only really hold for the second of the examples, the Dualgruppe constituted of modules, therefore I want to name it Modulgesetz, and any Dualgruppe verifying these laws, I want to name Dualgruppe of the module type. [Dedekind, 1897, 115]

\section{Conclusion}

Being able to provide a rigorous presentation of a theory, concept or result was a strong incentive in Dedekind's works. Such an incentive could be seen as a rigorist approach, opposed to creativity in mathematics. However, in Dedekind's mathematics, attempts at providing a more rigorous theory played a pivotal role in the introduction of new concepts and methods, from cuts to the arithmetization of Riemannian function theory.

Extending [Detlefsen, 2012], the main aspects of Dedekind's ideal of rigor, articulated around what Detlefsen coined the Dedekind principle, can be summed up in the following criteria:

- Avoidance of any kind of intuition or so-called evidence.

\footnotetext{
${ }^{37}$ The drafts suggest that Dedekind was significantly less interested in this law than he was in the Modulgesetz. He only occasionally considers ideals and the Idealgesetz.
} 
- Founding the theory on unprovable premisses, or at least well-defined notions, so as to avoid surreptitious assumptions and gaps in deductions.

- Definition and methods of proof should cover all possible cases (ideally, one definition for all cases). The theory must not be developed on the basis of a priori hypotheses or particular properties (including Darstellungformen). Special cases should be avoided.

- The theory should provide tools to find new results and new proofs without having to use external tools. ${ }^{38}$

Dedekind also presented a rigorous approach as being necessary for his understanding of mathematics. In addition, he made a clear distinction between the path to invention of a concept or result and the way he chose to define or present said concept or result. Both these aspects of Dedekind's reflections on his mathematical practice led me to consider what kind of rigor supported his mathematics in the making, as observable in his mathematical drafts - in the "back" of mathematics, as Hersch put it.

The analysis of draft researches of what would eventually become $D u$ algruppe theory showed, on the one hand, that Dedekind's research process relied on experimentations with computations and gradual generalizations, which allow him to observe the (potential) laws, and are more akin to an inductive than a deductive approach. In particular, Dedekind's use of examples, particular cases and step-wise generalization departs from his requirement of generality. This seems to be a difficulty for the principles of Dedekindian rigor identified in his publications, for they would not admit any sort of empirical evidence, nor would they accept an inductive approach as a rigorous one.

While assuming that 'rigor' could be a pre-requisite of mathematical reasoning would be naive, it has certainly become a pre-requisite of communicating one's results to other mathematicians. Does this imply that the Dedekindian ideal of rigor is essentially a principle on how to write good mathematics? This might be too reductive, considering the importance given to rigor by Dedekind. At the very least, it is an ideal that guides how (and sometimes why) Dedekind wants to work, especially insofar as, for him, rigor is presented as a key component of his mathematical understanding. In that case, should such researches be dismissed as being of little epistemic

\footnotetext{
${ }^{38}$ For example, being able to treat questions "related to continuity", that is, topological questions, with the algebraico-arithmetical arsenal developed in [Dedekind and Weber, 1882].
} 
significance? Their sheer volume in Dedekind's Nachlass would suggest the opposite. In fact, the kind of ampliative reasoning that appears in his systematic explorations of the properties of modules could be seen as a way to work with his intuitions of module-theoretical properties, so as to verify and prove them, and hence eliminate intuition and eventually obtain a rigorous presentation.

On the other hand, I put forward the trial-and-error aspects of Dedekind's research practices, by studying the path of a specific law, the Modulgesetz. I showed that it took many such trials, many attempts at providing an accurate proof, and many steps in the clarification of the property, for its status in the theory to stabilize. Interestingly, through these processes, some of Dedekind's principles of rigor are effectively put into action. Dedekind identified the fundamental laws of the operations + and - (sometimes designated as $\varphi$ and $\psi$ ), and aimed at proving properties (notably the Modulgesetz) based on these laws alone. Dedekind also aimed at proving everything that was provable, using a systematic, quasi-combinatorial approach. If the trajectory of the Modulgesetz is an example of Dedekind's attempts at applying his own ideal of rigor, it also shows how important setting up the appropriate conceptual frame is, for its actual application. Related to this is the question of the role played by the ideal of rigor in mathematical practice. In addition to being a motive for some researches, Dedekind's drafts suggest that it could also play a creative role in mathematics, much in the sense that logic or axiomatics did for authors such as Leibniz, Hilbert or Robinson (see [Benis-Sinaceur, 1989]).

From the drafts, it appears that rigor intervenes on two levels in these preliminary researches: regulating mathematical practice in the first steps of research, and more purposely in a step of rigorization, likened to verifying and justifying the results, and anterior to the textualization. Dedekindian rigor, as studied in [Detlefsen, 2012, Detlefsen, 2018] is thus gradually set up. The deductive hierarchy that constitutes the core of his ideal of rigor is an aspect that emerges progressively, as what Dedekind considers the appropriate way to formulate definitions, theorems, etc., after analysing, generalizing, verifying mathematical experimentations and explorations. Additional studies, such as a critical analysis of the genesis of Was sind und was sollen die Zahlen? (a work currently in progress), will help clarifying to what extent rigorization is a step in the research process, and if such a step should be distinguished from a certain rigor ingrained in mathematical practice.

What is clear, after the analysis of Dedekind's drafts, is that the genesis of rigorous mathematics does not necessarily hold up to the same standards of rigor. It could be tempting to dismiss all the preliminary researches as 
mere poking around in the dark, but that would dismiss a large part of the actual research process. It could also be tempting to try to modify or extend - but maybe at the same time loosen - our description of Dedekindian rigor enough so that it could uniformly fit all aspects of his work. I believe that none of these solutions are fruitful, and that the observations made by studying Dedekind's drafts should prompt us to think about mathematical rigor as a manifold, complex issue that requires nuanced descriptions accounting for its diversity - even in the works of a single mathematician.

\section{References}

[Aleksandrov, 1963] Aleksandrov, A. D. (1963). A General View of Mathematics. In Aleksandrov, A. D., Kolmogorov, A. N., and Lavrent'ev, M. A., editors, Mathematics: its Content, Methods and Meaning. English translation by S. H. Gould, K. A. Hirsch and T. Bartha. Cambridge, Massachusetts: M.I.T. Press.

[Benis-Sinaceur, 1989] Benis-Sinaceur, H. (1989). Ars inveniendi aujourd'hui. Les Études philosophiques, pages 199-214.

[Corry, 2004] Corry, L. (2004). Modern Algebra and the Rise of Mathematical Structures. Springer, New York.

[Dedekind, 1871] Dedekind, R. (1871). Über die Composition der binären quadratischen Formen, Xth Supplement. In Vorlesungen über Zahlentheorie, 2nd edition, pages 380-497. Partiellement In [Dedekind, 1932], volume III, p. 223-262.

[Dedekind, 1872] Dedekind, R. (1872). Stetigkeit und irrationale Zahlen. In [Dedekind, 1932], volume III, pages 315-335. Transl. in [Ewald, 2005], pages 765-778. All references to this translation.

[Dedekind, 1877a] Dedekind, R. (1876-1877a). Théorie des nombres entiers algébriques. Publié en cinq parties dans le Bulletin des Sciences Astronomiques et Mathématiques: BSAM 11 (1876), p. 278-288; BSAM Sér. 2(1):1 (1877), p. 17-41, 69-92, 144-164, et 207-248. Partiallt repr. in [Dedekind, 1932], III:262-296. Transl. in [Stillwell, 1996]. All references to this translation.

[Dedekind, 1877b] Dedekind, R. (1877b). Über die Anzahl der IdealKlassen in den verschiedenen Ordnungen eines endlichen Körpers. In [Dedekind, 1932], volume I, pages 105-159. 
[Dedekind, 1879] Dedekind, R. (1879). Über die Theorie der algebraischen Zahlen, XIth Supplement. In Vorlesungen über Zahlentheorie, 3e édition, pages 434-611. Partiellement In [Dedekind, 1932], volume III, pages 297315 .

[Dedekind, 1888] Dedekind, R. (1888). Was sind und was sollen die Zahlen? Braunschweig, Vieweg. In [Dedekind, 1932], volume III, pages 335-392. Transl. in [Ewald, 2005], pages 787-832. All references to this translation.

[Dedekind, 1894] Dedekind, R. (1894). Über die Theorie der algebraischen Zahlen, XIth Supplement. In Vorlesungen über Zahlentheorie, 4e édition, pages 434-657. In [Dedekind, 1932] III, p. 1-222.

[Dedekind, 1897] Dedekind, R. (1897). Über Zerlegungen von Zahlen durch ihre größten gemeinsamen Teiler. In [Dedekind, 1932], volume II, pages $103-147$.

[Dedekind, 1900] Dedekind, R. (1900). Über die von drei Moduln erzeugte Dualgruppe. In [Dedekind, 1932], volume II, pages 236-271.

[Dedekind, 1932] Dedekind, R. (1930-1932). Gesammelte mathematische Werke. 3 vols. Robert Fricke, Emmy Noether et Öystein Ore, Éditeurs, F. Vieweg and Sohn, Vieweg, Braunschweig. Re-éd. (1968) Chelsea Publishing Co., New York.

[Dedekind, 2008] Dedekind, R. (2008). La Création des Nombres. Vrin, Paris.

[Dedekind and Weber, 1882] Dedekind, R. and Weber, H. (1882). Theorie der algebraischen Funktionen einer Veränderlichen. In Journal für reine und angewandte Mathematik, Bd. 92, S. 181-290. In [Dedekind, 1932], volume I, pages 238-351. Trad. en. in [Stillwell, 2012]. All references to this translation.

[Detlefsen, 2012] Detlefsen, M. (2012). Dedekind against intuition: rigor, scope and the motives of his logicism. In Cellucci, C., Grosholz, E., and Ippoliti, E., editors, Logic and Knowledge, pages 205-217, Cambridge. Cambridge Scholars Publishing.

[Detlefsen, 2018] Detlefsen, M. (2018). "Weyl on proof, intuition and knowledge", invited lecture, Das Kontinuum: One hundred years later, congress honoring the centenary of the publication of Hermann Weyl's Das Kontinuum. School of Mathematics, University of Leeds,. 
[Edwards et al., 1982] Edwards, H., Neumann, O., and Purkert, W. (1982). Dedekind's 'Bunte Bemerkungen' zu Kroneckers 'Grundzüge'. Archive for History of Exact Sciences, 27(1):49-85.

[Edwards, 2010] Edwards, H. M. (2010). The Algorithmic Side of Riemann's Mathematics. In Bott, R. and Kotiuga, P., editors, A Celebration of the Mathematical Legacy of Raoul Bott, CRM proceedings \& lecture notes, Providence. American Mathematical Society.

[Ewald, 2005] Ewald, W. B. (2005). From Kant to Hilbert: A Source Book in the Foundations of Mathematics, 2 vols. Oxford University Press, Oxford.

[Grattan-Guinness, 2005] Grattan-Guinness, I., editor (2005). Landmark Writings in Western Mathematics 1640-1940. Elsevier Science.

[Haffner, 2014] Haffner, E. (2014). D'un point de vue rigoureux et parfaitement général : pratique des mathématiques rigoureuses chez Richard Dedekind. Philosophia Scientice, 18(1):131-156.

[Haffner, 2017] Haffner, E. (2017). Strategical use(s) of arithmetic in Richard Dedekind and Heinrich Weber's Theorie der algebraischen Funktionen einer Veränderlichen. Historia Mathematica, 44(1):31-69.

[Haffner, 2019] Haffner, E. (2019). From modules to lattices, insight into the genesis of Dedekind's Dualgruppen. British Journal for History of Mathematics, 34(1):23-42.

[Haffner, pear] Haffner, E. (to appear). Duality as a guiding light in the genesis of Dedekind's Dualgruppen.

[Hales, 2007] Hales, T. C. (2007). Jordan's Proof of the Jordan Curve Theorem. Studies In Logic, Grammar And Rhetoric, 10(23):45-60.

[Heijenoort, 1967] Heijenoort, J. V. (1967). From Frege to Gödel: A Source Book in Mathematical Logic, 1879-1931. Source books in the history of the sciences. Harvard University Press.

[Hersch, 1991] Hersch, R. (1991). Mathematics has a front and a back. Synthese, 8(2):127-133.

[Jacobi, 1881] Jacobi, C. G. J. (1881). Gesammelte Werke, Vol. I. Reimer. [Repr. New York: Chelsea, 1969.], Berlin. 
[Klein, 1895] Klein, F. (1895). Über Arithmetisierung der Mathematik. NG, Geschäftliche Mitteilungen., pages 965-971. Trad. fr. (dans Nouvelles annales de mathématiques, 16 (1897), 114-128.

[Kleiner, 1991] Kleiner, I. (1991). Rigor and Proof in Mathematics: A Historical Perspective. Mathematics Magazine, 64(5):291-314.

[Kronecker, 1882] Kronecker, L. (1882). Grundzüge einer arithmetischen Theorie der algebraischen Grössen. Journal für die reine und angewandte Mathematik, 92:1-222. In [Kronecker, 1899], p. 237-388.

[Kronecker, 1899] Kronecker, L. (1899). Werke, 3 volumes. Teubner. Re-éd. (1968) Chelsea, New York., Leipzig.

[Laugwitz, 2009] Laugwitz, D. (2009). Bernhard Riemann 1826-1866: Turning Points in the Conception of Mathematics. Modern Birkhäuser Classics. Springer, New York.

[Lejeune-Dirichlet, 1863] Lejeune-Dirichlet, J. P. G. (1863). [2e éd. 1871, 3e éd. 1879, 4e éd. 1894], Vorlesungen über Zahlentheorie. Vieweg und Sohn, Richard Dedekind, éditeur. Braunschweig.

[Mehrtens, 1979] Mehrtens, H. (1979). Die Entstehung der Verbandstheorie. Hildesheim, Gerstenberg.

[Pierpont, 1928] Pierpont, J. (1928). Mathematical Rigor, Past and Present. Bull. AMS, 34:23-53.

[Riemann, 1876] Riemann, B. (1876). Gesammelte mathematische Werke und wissenschaftlicher Nachlass. H. Weber and R. Dedekind (eds). Teubner, Leipzig. Repr. 1892. Repr. with Nachträge (1902), M. Noether and W. Wirtinger (eds), Teubner, Leipzig. Reéd. (1953), Dover, New York. Re-ed. (1990), Springer/Teubner, Berlin.

[Scheel, 2014] Scheel, K. (2014). Der Briefwechsel Richard Dedekind - Heinrich Weber. Abhandlungen der Akademie der Wissenschaften in Hamburg (5). De Gruyter Oldenbourg, Hamburg.

[Schlimm, 2011] Schlimm, D. (2011). On the creative role of axiomatics. The discovery of lattices by Schröder, Dedekind, Birkhoff, and others. Synthese, 183(1):47-68.

[Schröder, 1873] Schröder, E. (1873). Lehrbuch der Arithmetik und Algebra. B. G. Teubner. 
[Stillwell, 1996] Stillwell, J. (1996). Theory of Algebraic Integers, by $R$. Dedekind. Cambridge University Press, Cambridge.

[Stillwell, 2012] Stillwell, J. (2012). Theory of Algebraic Functions of One Variable. Transl. of [Dedekind and Weber, 1882]. American Mathematical Society, Providence.

\section{Acknowledgements}

This work was supported by a public grant as part of the Investissement d'avenir project, reference ANR-11-LABX-0056-LMH, LabEx LMH.

Part of this work was also done while working as a member of the Deutsche Forschungsgemeinschaft - DFG-Forschungsprojekt 'Dualität - ein Archetypus mathematischen Denkens' 279002986.

I would like to thank Paddy Blanchette and an anonymous referee for their insightful remarks on a first version of this paper. 